\title{
Síntesis, caracterización y aplicación de poli-3,4-dialcoxitiofenos
}

\author{
Sara Urrego Riveros ${ }^{1 \star}$, César Sierra Ávila ${ }^{2 \star \star}$, Oscar Rodríguez Bejarano ${ }^{1 \star \star \star}$ \\ ${ }^{1}$ Grupo de Electroquímica y Termodinámica Computacional \\ ${ }^{2}$ Grupo de Macromoléculas \\ Departamento de Química, Facultad de Ciencias, Universidad Nacional de Colombia, \\ Bogotá, Colombia
}

FeCha de entrega: Abril 12 De 2012
FeCha de Evaluación: Abril 30 DE 2012
Fecha de Aprobación: MAyo 3 DE 2012

\begin{abstract}
Resumen A pesar del rápido crecimiento que ha tenido el uso de las celdas solares para la generación de electricidad, los altos costos de fabricación de los dispositivos tradicionales ha impedido su masificación. Se hace necesario desarrollar celdas solares de menor costo. Una de las nuevas tecnologías que genera mayores expectativas es la de las celdas solares orgánicas, basadas en polímeros semiconductores, las cuales, hasta el momento, presentan eficiencias de conversión inferiores al $10 \%$. Presentamos aquí algunos resultados de un trabajo de investigación que busca la síntesis de nuevos polímeros semiconductores, específicamente de poli-3,4-diisobutoxitiofeno y poli-3,4-dimetoxitiofeno, con propiedades ópticas, electrónicas y estructurales adecuadas para la fabricación de celdas solares con mayores eficiencias de conversión.
\end{abstract}

Palabras Clave: celdas solares orgánicas, poli-dialcoxitiofenos, polímeros conductores.

\begin{abstract}
Despite the rapid growth that has gained the use of solar cells for electricity generation, high manufacturing costs of traditional devices has prevented their widespread implementation. Then, it is necessary to develop lower-cost solar cells. One of the technologies that generate greater expectations is the organic solar cell, semiconductor-based polymers, which so far have conversion efficiencies lower than $10 \%$. We present here some results of a research project that seeks the synthesis of new semiconducting polymers, specifically poly-3,4-diisobutoxythiophene
\end{abstract}

* Estudiante de Maestría en Ciencias - Química. surregor@unal.edu.co

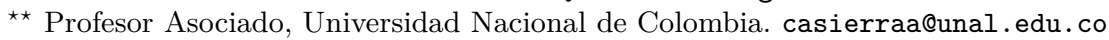

*** Químico, Dr. Sc. Profesor Asociado Departamento de Química Universidad Nacional de Colombia. Áreas de interés: Desarrollo de metodos voltamétricos de análisis para el monitoreo de iones metálicos. Desarrollo de nuevos métodos de síntesis de materiales semiconductores, los cuales incluyen CBD (Chemical Bath Deposition), electrodeposición e inkjet printing. Cálculo de diagramas de fases de algunos sistemas binarios con aplicaciones fotovoltaicas. orodriguez@unal.edu.co 
and poly-3,4-dimetoxythiophene with optical, electronic and structural properties appropriate for the manufacture of solar cells with higher conversion efficiencies.

Keywords: organic solar cells, poly-dialcoxythiophenes, conducting polymers.

\section{Introducción}

Durante los últimos 40 años las celdas solares basadas en materiales inorgánicos, tales como Si mono y policristalino, GaAs, CdTe, CuInSe 2 , etc., se han mostrado como una alternativa viable al problema de la crisis energética, puesto que utilizan una fuente energética renovable, gratuita y prácticamente inagotable, presentan altas eficiencias de conversión de energía radiante y su producción se realiza a escala industrial; sin embargo, los altos costos de manufactura, los efectos negativos de algunos de sus componentes sobre el medio ambiente y la escasez en la corteza terrestre de las materias primas para la producción de algunas de ellas, se presentan como serias limitantes para una mayor aplicación a nivel mundial. Un nuevo tipo de celdas solares basadas en polímeros semiconductores, como los poli-3,4-dialcoxitiofenos, se presentan como una nueva alternativa a las celdas solares tradicionales. A pesar de que en la actualidad aún tienen una eficiencia de conversión de energía radiante a eléctrica muy baja, las celdas solares basadas en polímeros semiconductores tienen una serie de ventajas: las materias primas son de fácil acceso y aunque son muy costosas, las cantidades utilizadas en cada celda son pequeñísimas; a diferencia de las inorgánicas, los procesos de fabricación son sencillos y de muy bajo costo, pues se pueden fabricar sobre sustratos flexibles y, dependiendo de la estructura química de los componentes, se pueden obtener módulos de diferentes colores, lo que las hace muy atractivas desde el punto de vista arquitectónico. De manera que la inclusión en el mercado de celdas solares, que sean más versátiles, flexibles y sobre todo económicas pueden propiciar un incremento notable en el uso de la energía solar, porque posiciona, en un futuro no muy lejano, a la energía solar fotovoltaica como la más promisoria para satisfacer la demanda energética de la humanidad, luego del agotamiento de los combustibles fósiles como fuente de energía.

\subsection{Celdas solares orgánicas}

Desde sus inicios las celdas solares orgánicas han evolucionado desde las más simples, las cuales solo hacen uso de un material altamente conductor que se encuentra entre dos electrodos, hasta llegar a las que han obtenido mejores resultados con respecto a su eficiencia de conversión eléctrica, construidas sobre la base del concepto de heterojuntura en el volumen (Bulk heterojunction o BHJ), en donde, la capa activa de la celda solar, es decir, la que absorbe la luz y genera los portadores de carga eléctrica, es una mezcla homogénea de un material donor de electrones (el polímero) con otro aceptor de electrones (en general un fulereno). Recientemente se han desarrollado nuevos tipos de celdas solares orgánicas, las 
cuales, incluyen un copolímero que presenta las funciones de ambos materiales donor y aceptor $[1,2]$.

Generalmente las celdas solares tipo BHJ están compuestas por un soporte transparente que puede ser flexible como el polietileno o rígido como el vidrio alcalino, y que no debe conducir la electricidad ni absorber la luz; sobre dicho sustrato se deposita una película delgada y transparente de un material que actúa como ánodo, el cual, generalmente es de óxido de estaño dopado con Indio (ITO por sus siglas en inglés), o de óxido de estaño dopado con Flúor (FTO por sus siglas en inglés). En algunos tipos de celda, sobre el ánodo se deposita un material orgánico altamente conductor, el cual, usualmente es una mezcla de poli-3,4-etilendioxitiofeno y poli-estireno sulfonato de Sodio (PEDOT:PSS, por sus siglas en inglés), y se denomina capa buffer al actuar de manera similar como lo hacen sus equivalentes en las celdas solares inorgánicas. Sobre la película de PEDOT:PSS se deposita la capa activa. La capa activa es una película delgada (con un espesor de $100 \mathrm{~nm}$ aprox.) y está compuesta por una mezcla homogénea de un donor y un aceptor de electrones. La máxima eficiencia de conversión reportada para una celda solar de este tipo, usa como capa activa la mezcla poli-3-hexiltiofeno, el donor de electrones, y un derivado del fullereno, el éster metílico del ácido fenil-C61-butirico (PCBM, por sus siglas en inglés) que actúa como aceptor de electrones. En esta capa es donde ocurre el proceso de absorción de luz, la generación de portadores de carga eléctrica, la separación de los mismos y su trasporte de carga hacia los electrodos. En su estructura más básica este tipo de celdas termina con una película delgada del material que hace las veces de cátodo o contacto eléctrico superior, el cual, generalmente es aluminio metálico. En la figura 1 se observa la sección trasversal de celdas tipo BHJ [1,3,4].

Tanto la capa activa como la buffer se depositan mediante una técnica denominada spin-coating en la que una solución de PEDOT:PSS o de los componentes de la capa activa, se agrega gota a gota sobre el sustrato que está en rotación a altas velocidades, encontrándose todo el sistema a baja presión y en una atmósfera libre de oxígeno $\left(\mathrm{Ar}\right.$ o $\left.\mathrm{N}_{2}\right)$ [1,3].

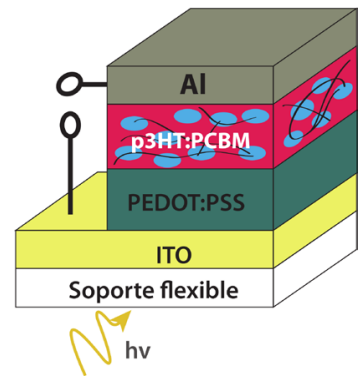

Figura 1. Sección transversal de una celda solar con estructura tipo BHJ. 


\subsection{Principio de operación de las celdas solares orgánicas}

Este tipo de celdas utiliza compuestos orgánicos semiconductores, los cuales, al presentar una diferencia entre el HOMO y LUMO dentro de su configuración, actúan de manera similar a las bandas de valencia y conducción en los semiconductores inorgánicos tipo $\mathrm{p}$ y $\mathrm{n}$.

En la figura 2 se muestra un esquema simplificado del diagrama de bandas de energías de la celda solar: cátodo, material donor de $\mathrm{e}^{-}$(D), material aceptor de $\mathrm{e}^{-}$(A) y ánodo. Se observa que en las CSO, la absorción de fotones por parte de la capa activa genera una transición electrónica entre estados HOMO y LUMO del material donor, generando un sistema de pares electrón-hueco conocidos como excitones, localizados en la estructura del compuesto; los exitones generados se difunden hasta la interface con el material aceptor donde se disocian generando portadores de carga libres (en el esquema: huecos, círculos rojos y electrones, círculos negros) que se mueven independientemente a través de cada material hasta llegar a los contactos eléctricos. Para lograr la separación de las cargas es necesaria la existencia de un campo eléctrico, que es proporcionado por la asimetría de la energía de ionización y función-trabajo de los materiales que actúan como electrodos. Esta asimetría es la razón del porqué el flujo de electrones se ve favorecido desde el electrodo con menor función-trabajo hacia el electrodo con mayor función trabajo (polarización directa) $[4,5]$.

Estas celdas solares presentan como inconveniente fundamental la existecia de procesos de recombinación del excitón, ya sea porque puede que no alcance a llegar a la interface, generando dentro del material donor procesos de luminiscencia por recombinación geminal o puede ser que se recombinen los huecos y electrones disociados en la interface, ya que al estar muy cerca éstos no alcancen a difundirse en sus respectivos materiales y generen también un efecto luminiscente dentro de toda la mezcla.

\subsection{Propiedades de los componentes de las celdas solares orgánicas}

Las propiedades de mayor interés de los semiconductores poliméricos tipo n es que transportan carga en tiempos muy rápidos (en el orden de los fs) y presentan altas constantes dieléctricas que permiten estabilizar el polaron formado luego de la disociación del excitón; estas constantes dieléctricas provienen de la deslocalización de la carga del sistema $\pi$. Los fullerenos son los sistemas más utilizados como material tipo n, debido a que aparte de las propiedades mencionadas previamente, también tienen LUMO's bajos que se combinan muy bien con los orbitales de los semiconductores tipo $\mathrm{p}[1,3]$.

Por otra parte los semiconductores tipo p, deben ser altamente conjugados y presentar así muy buena conductividad eléctrica y alta movilidad de huecos; deben poseer unos anchos de banda prohibida (Band Gap), $E_{g}$, pequeños $\left(E_{g}<2\right.$ $\mathrm{eV}$ ) para que puedan absorber fotones en la mayor porción posible del espectro de radiación; debido a los métodos que se emplean para obtener las capas activas en las CSO, estos compuestos deben ser muy solubles en los solventes comunmente utilizados en la etapa de deposición (spin-coating, inkjet printing, etc.). Los 


\section{Donor}

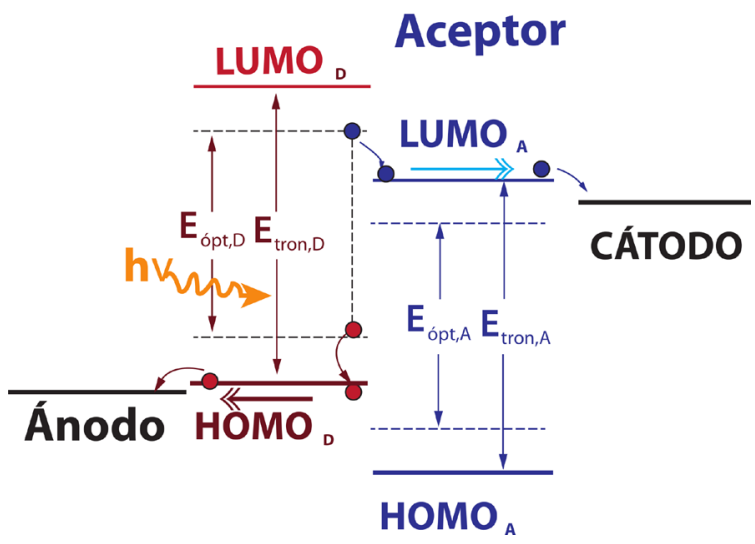

Figura 2. Procesos que ocurren dentro de la capa absorbente de una celda solar orgánica.

polímeros que generalmente son utilizados como este tipo de material son los derivados del tiofeno. En el caso de los tiofenos monosustituidos, se introduce el concepto de ordenamiento regio regular cuya geometría orienta a los anillos del tiofeno en un mismo plano, permitiendo un aumento en la conjugación y deslocalización de enlaces $\pi$; este efecto se incrementa al utilizar azufre como hetero átomo permitiendo una fácil ionización y formación del excitón y por consiguiente un transporte de carga más eficiente.

En la figura 3 se observan los diferentes regioisómeros de los polímeros derivados del tiofeno, el regioisómero que permite una mayor conjugación es el de acoplamiento HT-HT (Head to Tail o cabeza-cola), es decir, que los tiofenos se enlazan a través de las posiciones 2,5 con geometría trans; esta configuración evita que hayan impedimentos estéricos de los sustituyentes entre sí y con el Azufre $[6,7,8]$.

Para cubrir la máxima absorción de radiación solar, se puede modificar el $E_{g}$ variando la distancia energética entre el HOMO y el LUMO. Esto puede ocurrir al aumentar su conjugación para que se facilite la deslocalización electrónica, lo cual, puede lograrse al sustituir los $\mathrm{H}$ en las posiciones 3 y 4 del tiofeno por grupos alquilo o alcoxi, logrando generar únicamente uniones HT-HT, obteniéndose así polímeros totalmente regioregulares y planos. Si se utilizan grupos alcoxi, el oxígeno al ser donor de electrones, estabiliza el intermediario de la polimerización y aumenta la energía del HOMO, disminuyendo así el $E_{g}$; además, si se realiza una doble sustitución, la polimerización del monómero va a producir polímeros totalmente regio regulares y planos tal como se mencionó previamente $[6,7,9]$.

Si bien las propiedades descritas son ideales para la utilización de los mencionados materiales para la fabricación de celdas solares, esto no quiere decir 
<smiles>[R]c1c(-c2sc(C(C)(C)C)c([R])c2[R])sc(-c2sc(C(C)(C)C)c([R])c2[R])c1[R]</smiles>

HT-HT

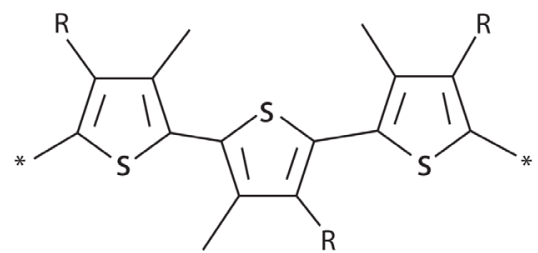

TT-HT<smiles>[R]c1c(-c2sc(-c3sc(C)c(C)c3C)c([R])c2C)sc(C)c1C</smiles>

$\mathrm{HT}-\mathrm{HH}$

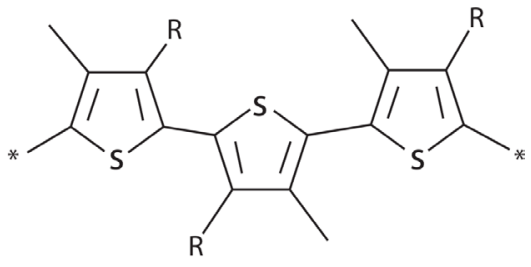

HH-TT

Figura 3. Disposición de los politiofenos monosusituidos en la posición 3.

necesariamente que solamente se puedan emplear para ello. En algunos casos y dependiendo de sus propiedades fotoluminiscentes pueden llegar a ser útiles también como materiale absorbentes en otro tipo de dispositivos llamados diodos emisores de luz orgánicos (OLED's por sus siglas en inglés).

El objetivo fundamental del trabajo de investigación aquí presentado, fue el de realizar la síntesis de polímeros regioregulares, derivados de tiofeno con anchos de banda prohibida, $E_{g}$, menores que $2 \mathrm{eV}$ y con bajos procesos de recombinación, es decir, que sean muy poco fluorescentes para poderlos utilizar como componentes de capas activas de celdas solares orgánicas. La estructura de los monómeros de partida para la obtención de los politiofenos regioregulares se muestra en la figura 4 .
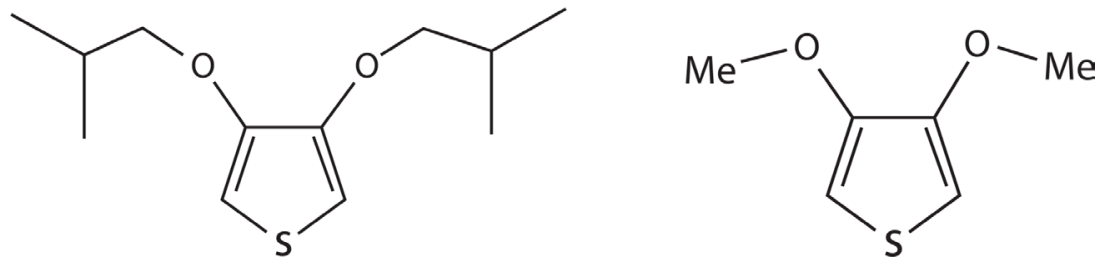

Figura 4. Estructura de los monómeros a estudiar y polimerizar (a) 3,4-dimetoxitiofeno (b) 3,4-diiisobutoxitiofeno. 


\section{Metodología}

La ruta de síntesis empleada, se puede observar esquematizada en la figura 5.

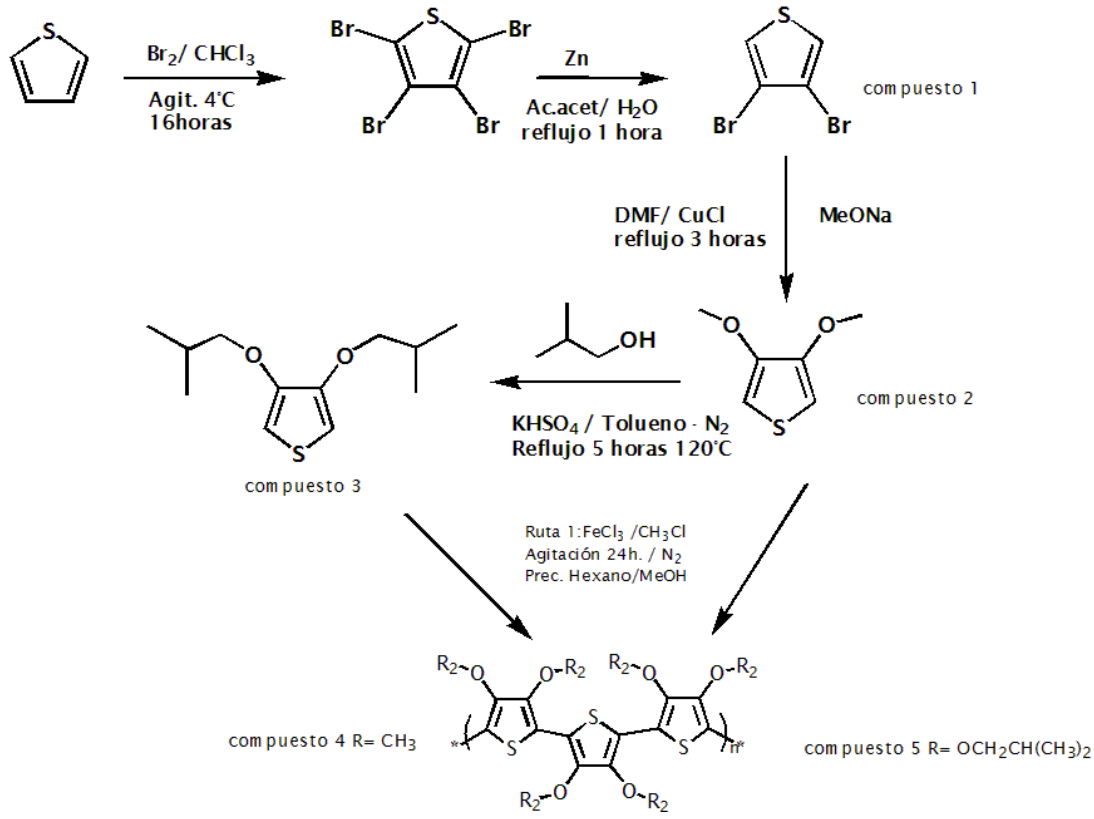

Figura 5. Ruta de síntesis empleada.

\subsection{Síntesis de 3,4-dibromotiofeno (compuesto 1 )}

En un balón de dos bocas se coloca el tiofeno (0,125 moles, $10 \mathrm{~mL}$ ) y el cloroformo (7 mL), se mantiene en agitación en un baño de hielo-agua; en una de las bocas del balón se conecta una trampa de vacío y ésta a su vez a una de $\mathrm{NaOH}$, para neutralizar el $\mathrm{HBr}$ formado y evitar así su fuga.

Por otro lado mediante un embudo de adición con regulador de presión, se adiciona lentamente el Bromo $(30 \mathrm{~mL})$ durante 14 horas. Al transcurrir este tiempo, se retira el embudo y se coloca un refrigerante para realizar un reflujo durante 2 horas. Al finalizar el tiempo, se detiene el reflujo y la mezcla de reacción se añade a una solución concentrada de $\mathrm{NaOH}$ para neutralizar el Bromo; la mezcla de reacción se deja en agitación durante toda la noche.

Al día siguiente se filtra el sólido obtenido y se deja secando. Mientras esto ocurre en un balón de dos bocas, se acondiciona un refrigerante y se colocan 39 $\mathrm{mL}$ de ácido acético, $50 \mathrm{~mL}$ de agua y 37, $42 \mathrm{~g}$ de $\mathrm{Zn}$ en polvo, se calienta hasta 
ebullición y se mantiene bajo un reflujo. Seguido de esto, lentamente se agrega el sólido seco sintetizado previamente (2,3,4,5-tetrabromotiofeno), teniendo en cuenta que por cada adición que se realice se debe esperar un tiempo prudencial para evitar que haya una sobrepresión. Al terminar de agregar el sólido se deja durante 10 minutos en ebullición y se procede a filtrar el líquido amarillo. La purificación del líquido se realiza mediante extracción líquido-líquido utilizando diclorometano como eluyente [10]. El 3,4-dibromotiofeno sintetizado es un líquido amarillo viscoso, presentó un rendimiento global de $69,7 \%$ y presentó un $\mathrm{Pe}=212$ ${ }^{\circ} \mathrm{C}$, los resultados del análisis espectroscópico fueron: FT-IR $\left(\mathrm{cm}^{-1}\right) 3109,2924$, $1474,1397,1096,785, d_{H}\left(400 \mathrm{MHz}^{-C_{C l}}\right) 7.328(2 \mathrm{H}, \mathrm{s}, \mathrm{CH}=\mathrm{C}) . d_{C}(100 \mathrm{MHz}$ $\left.\mathrm{CDCl}_{3}\right) 113.942,123.747$.

\subsection{Síntesis de 3,4-dimetoxitiofeno (compuesto 2)}

Primero se realiza la síntesis in-situ del metóxido de sodio, por lo que en un balón de tres bocas, se colocan $39 \mathrm{~mL}$ de metanol y se calienta externamente, manteniéndose bajo atmósfera inerte de nitrógeno; luego suavemente se adicionan pequeñas cantidades de Sodio 3, $20 \mathrm{~g}$ (0,14 moles), teniendo en cuenta que entre cada adición, se debe esperar a su disolución para así evitar una sobrepresión.

Por otra parte se prepara una solución de 27,1 mmol de compuesto 1 en $25 \mathrm{~mL}$ de dimetilformamida (DMF) colocándose en un embudo de adición; por aparte en un adicionador de sólidos se colocan 0,33 moles de $\mathrm{CuCl}$, seco.

Al terminar de formarse el metóxido de sodio la solución del compuesto 1 y el $\mathrm{CuCl}$ se adicionan lentamente, y se mantienen bajo atmósfera inerte, con un reflujo de $90{ }^{\circ} \mathrm{C}$ y por un lapso de tiempo de 12 horas, tiempo en el cual se realizan controles con cromatografía en capa delgada (CCD).

$\mathrm{Al}$ terminar el tiempo de reacción y asegurar la presencia del producto por CCD, la mezcla de reacción se vierte sobre una mezcla agua-hielo acidificada con $\mathrm{HCl}$. Al diluirse el hielo la mezcla es llevada a un embudo de separación donde se realizan lavados con diclorometano, formándose una capa acuosa color verde y el producto que es amarillo-naranja, en la capa orgánica [11].

El rendimiento de la reacción fue de 55,04\%, el Pe $=250-252{ }^{\circ} \mathrm{C}$. Los resultados del análisis espectroscópico fueron: FT-IR $\left(\mathrm{cm}^{-1}\right)$ 3116.97, 2947.23, $1674.21,1504.48,1411.89,1211.30,1141.86,995.27,856.39,756.11,501.49 . \mathrm{RMN}$ $d_{H}\left(400 \mathrm{MHz}^{\mathrm{CDCl}} 3\right) 8.990(2 \mathrm{H}, \mathrm{s}, \mathrm{S}-\mathrm{CH}=\mathrm{C}), 6.655\left(6 \mathrm{H}, \mathrm{s}, \mathrm{O}-\mathrm{CH}_{3}\right), d_{C}(100$ $\mathrm{MHz} \mathrm{CDCl}_{3}$ ) 155.296, 103.764, 64.981.

\subsection{Síntesis de compuesto 3}

A partir de 4,13 mmol del compuesto 2, $2 \mathrm{~mL}$ de tolueno, 169 mmoles de alcohol isobutílico $(1,25 \mathrm{~mL})$, se colocan en un balón de dos bocas, adaptado a un refrigerante y se mantiene bajo una adecuada atmósfera de Nitrógeno sin fugas. El tiempo de reacción se determinó mediante control por CCD. Luego de pasado el tiempo de reacción el precipitado se filtra y el sobrenandante oscuro aceitoso fue el 3,4-diisobutoxitiofeno [12]. El rendimiento de la reacción fue de 46,73\%, el $\mathrm{Pe}=250-252{ }^{\circ} \mathrm{C}$, los resultados del análisis espectroscópico fueron: FT-IR 
$\left(\mathrm{cm}^{-1}\right)$ 2958.8, 2854, 1500, 1465.9, 1373.3, 1203.58, 1141.86, 740.67. RMN $d_{H}$ $\left(400 \mathrm{MHz} \mathrm{CDCl}_{3}\right) 6.163(2 \mathrm{H}, \mathrm{s}, \mathrm{S}-\mathrm{CH}=\mathrm{C}), 3.867\left(4 \mathrm{H}, \mathrm{t}, \mathrm{O}-\mathrm{CH}_{2}-\mathrm{C}\right), 2.36(1 \mathrm{H}$, $\left.\mathrm{s}, \mathrm{CH}-\left(\mathrm{CH}_{3}\right)_{2}\right), 1.804\left(6 \mathrm{H}, \mathrm{q}, \mathrm{CH}_{3}\right), d_{C}\left(100 \mathrm{MHz}_{\mathrm{CDCl}}\right)$ 147.3, 96.5, 69.9, $30.76,18.9,13.5$. о

\subsection{Polimerización oxidativa del compuesto 2, poli-3,4-dimetoxi- tiofeno (compuesto 4)}

La polimerización oxidativa del compuesto 2 se realiza a partir de una solución de $1 \mathrm{~mol}$ del monómero y $12 \mathrm{~mL}$ de cloroformo, por aparte se prepara una solución de $1 \mathrm{mmol} \mathrm{FeCl}_{3}$ en $10 \mathrm{~mL}$ de cloroformo, las dos soluciones se llevan a un balón de dos bocas, y se dejan agitando a temperatura ambiente bajo reflujo. En el transcurso de la reacción se realiza control por CCD para poder terminarla al desaparecer el monómero. Al ocurrir esto se agrega 1,2 mL de hidracina y se deja en agitación durante toda la noche. El sólido obtenido es filtrado y lavado con hexano, y se mantiene en un soxlet durante varios días. Luego el sólido de color violeta es filtrado sobre silica 60, realizando lavados con acetato de etilo [12]. El poli-3,4-dimetoxitiofeno obtenido presentó como resultados espectroscópicos los siguientes: FT-IR (cm ${ }^{-1}$ ) 2939-2839 (banda ancha), 1458, 1250, 1165, 1064, 810. RMN dH (400 MHz $\left.\mathrm{CDCl}_{3}\right)$ 3.903, 0.884-1.650.

\subsection{Polimerización oxidativa del compuesto 3 (compuesto 5)}

La polimerización se llevó a cabo con las mismas cantidades mostradas para la síntesis del compuesto 4 pero con la diferencia que se realizó bajo atmósfera inerte de $\mathrm{N}_{2}$ y en un baño de agua-hielo. La extracción se realizó primero con metanol para retirar las sales inorgánicas formadas y luego con hexano para retirar el monómero que no reaccionó. El sólido obtenido fue filtrado sobre sílica, realizando lavados con cloroformo [13]. El poli-3,4-diisobutoxitiofeno presentó los siguientes resultados espectroscópicos: FT-IR $\left(\mathrm{cm}^{-1}\right)$ 2924, 1457, 1373, 1265, 1026, 802, 740. RMN $d_{H}\left(400 \mathrm{MHz} \mathrm{CDCl}_{3}\right)$ 4.187, 2.036, 1.548, 1.381, 1.221, 1.076 .

\subsection{Polimerización electroquímica del compuesto 3}

La polimerización electroquímica se hizo con el fin de realizar una deposición más homogénea y directa del polímero semiconductor tipo p, sobre los sustratos utilizados en las celdas solares, que en este caso son de vidrio alcalino recubierto con FTO.

La celda electroquímica empleada, estaba constituida por tres electrodos en donde, como pseudo-electrodo de referencia, se utilizó un alambre de Ag (PRE $\mathrm{Ag} / \mathrm{Ag}^{+}$), como contra-electrodo: un alambre de Pt, y como electrodo de trabajo: FTO soportado sobre vidrio con una ligera capa de oro. En la figura 6 se puede apreciar un esquema de la celda electroquímica utilizada. 


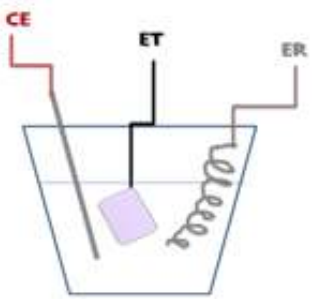

Figura 6. Disposición de la celda electroquímica utilizada para la electrosíntesis del poli3,4-dimetoxitiofeno. CE- contraelectrodo: Pt, ET- electrodo de trabajo: vidrio recubierto con FTO con una capa delgada de oro, ER- electrodo de referencia: pseudo-electrodo de Ag.

El electrodo de trabajo fue sometido previamente a una limpieza exhaustiva que incluía, lavados con detergente no iónico, agua desionizada, solución sulfocrómica y finalmente otro lavado con agua desionizada. Cada lavado realizado, se completaba colocando el sistema electrodo-solución en ultrasonido durante cinco minutos.

Luego de esto, se formó la capa delgada de oro la cual se hizo en una celda electroquímica, utilizando como electrodo de referencia $\mathrm{Ag} / \mathrm{AgC}$ sobre una solución de $\mathrm{Au}$ (III) 96 ppm y aplicando un potencial constante de $-20 \mathrm{mV}$ durante $240 \mathrm{~s}$. Por otra parte el contraelectrodo fue limpiado suavemente por abrasión con $\gamma$-alúmina.

En la celda electroquímica, se colocó una solución en acetonitrilo, 0,1 M en monómero y 0,1 $\mathrm{M}$ en $\mathrm{LiClO}_{4}$, este último utilizado como electrolito soporte. La electropolimerización se realiza por dos métodos: el primero consiste en aplicar 200 ciclos de potencial de $900 \mathrm{a}-60 \mathrm{mV}$ con velocidades de barrido de 25 y 50 $\mathrm{mV} / \mathrm{s}$; y en el segundo se aplican 200 escalones de potencial de 80 a $480 \mathrm{mV}$ con una velocidad de barrido de $250 \mathrm{mV} / \mathrm{s}$.

\section{Resultados y discusión}

Las señales en el FT-IR al ser diferentes en los productos de cada reacción de la ruta, ayudan a llevar un control de reacción debido a la presencia o ausencia de señales. Una de las señales más importantes es la de las flexiones $\mathrm{C}=\mathrm{C}-\mathrm{S}$ la cual se presenta entre $720-780 \mathrm{~cm}^{-1}$ y aparece en todos los productos incluido los polímeros pero con diferente intensidad [9].

Como prueba de la sustitución nucleofílica del $\mathrm{MeO}^{-}$sobre el $\mathrm{Br}$ en el anillo aromático está la presencia de señales características entre $1160-1030 \mathrm{~cm}^{-1}$ que son debidas a flexiones entre $\mathrm{C}-\mathrm{O}-\mathrm{C}$, las cuales están presentes en los compuestos 3 y 4 excepto en el 2 (3,4-dibromotiofeno).

Mediante está caracterización no se puede determinar si la polimerización de los monómeros se desarrolló completamente, debido a que las señales de los 
polímeros coinciden con la de los monómeros, presentando solamente diferente intensidad.

Para poder identificar los polímeros sintetizados, se utilizó el ${ }^{1} \mathrm{H}$ RMN, en el cual se muestra la ausencia de los protones de las posiciones 2 y 5 del tiofeno, los cuales presentan un desplazamiento de 8,26 pmm para el compuesto 3 y 6,86 ppm para el compuesto 4. Los espectros de los respectivos polímeros pueden observarse en las figuras 7 y 8 . Respecto al compuesto 4 no se puede asegurar completamente la estructura con este tipo de espectroscopia, y pareciera que existiera una mezcla entre el 3,4-dimetoxitiofeno y el 3,4-diisobutoxitiofeno.

En estos espectros RMN protónicos de los polímeros (figuras 7 y 8) se puede observar que algunas señales están presentes campos más altos con respecto al de sus monómeros, debido a que la estructura del polímero contiene un gran número de grupos. Este es el caso de los protones del $\mathrm{MeO}^{-}$del compuesto 3 los cuales aparecen con un $\delta 6,5 \mathrm{ppm}$ y al polimerizar aparecen a $3,5 \mathrm{ppm}$. Con el espectro de este polímero, también se puede observar la presencia de señales no deseadas $(\delta 0,884,1,242$ y 1,650 ppm), las cuales son debido a un polímero de bajo peso molecular, sin embargo no se ha explicado con exactitud de qué parte del polímero provienen.

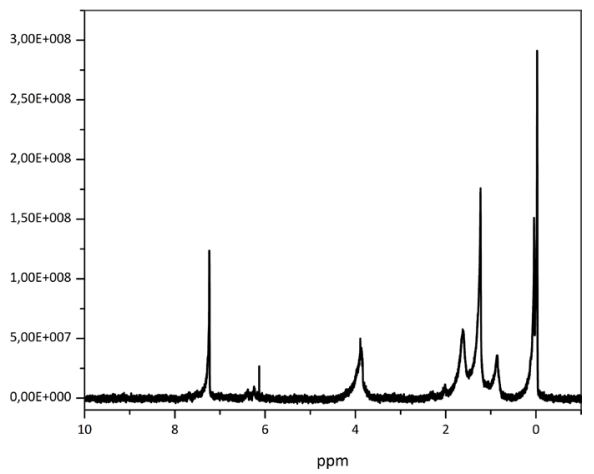

Figura 7. Espectro ${ }^{1} \mathrm{HRMN}$ del poli-3,4-dimetoxitiofeno.

En las figuras 9 y 10, se observan los espectros UV-Vis obtenidos para determinar el band gap (diferencia entre el HOMO-LUMO) de los polímeros; este se calcula a partir del corte que realiza la tangente del punto de inflexión de la banda de menor energía [14].

Además de este experimento se determinó mediante espectroscopía de fluorescencia que ninguno de los dos polímeros sintetizados presentan fluorescencia (resultados no mostrados aquí). El hecho de que no presenten fluorescencia y que tengan un band gap entre 2, 10 y 1,97 eV, como se observa en las figuras 9 y 10 respectivamente, hace pensar que los polímeros bajo estudio absorben radiación en un rango apreciable del espectro de luz solar. Una vez absorbido, un fotón de 


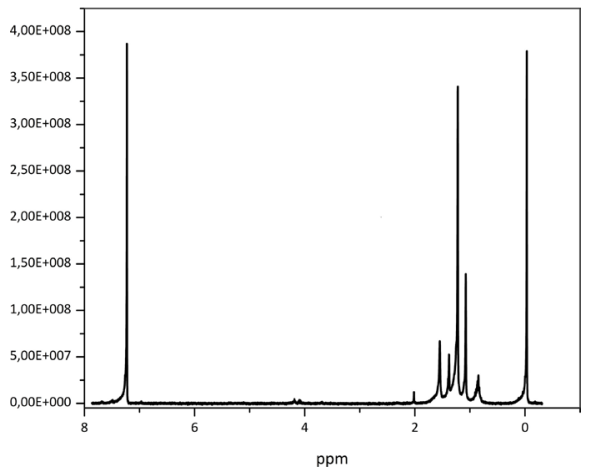

Figura 8. Espectro ${ }^{1} \mathrm{HRMN}$ del poli-3,4-diisobutoxitiofeno.

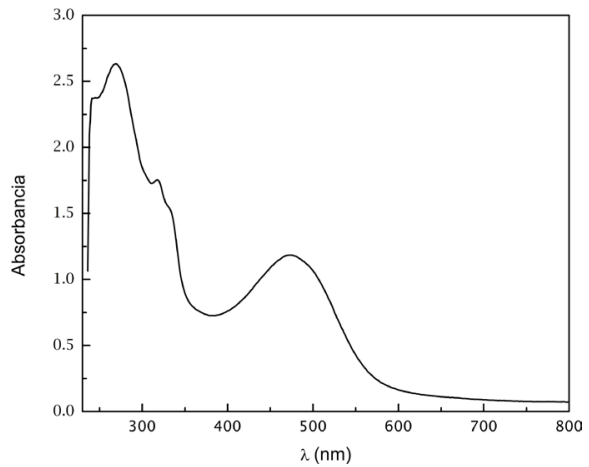

Figura 9. Espectro UV-Vis del poli-3,4-dimetoxitiofeno.

luz tiene la energía suficiente para generar el excitón dentro de los polímeros y según los resultados, es posible que no se recombine, debido a que los polímeros no presentan procesos radiativos en forma de fluorescencia. Por lo tanto de acuerdo a estas propiedades, se puede decir que tanto el poli-3,4-dimetoxitiofeno como el poli-3,4-diisobutoxitiofeno, presentan dos de las características más importantes para ser utilizados como semiconductores tipo p en una celda solar orgánica.

Se puede añadir estos dos polímeros son muy solubles en los solventes orgánicos que se utilizan regularmente en la obtención de las capas activas de las CSO, como son el cloroformo y el diclorobenceno; esta propiedad le da una ventaja potencial adicional al uso de estos dos polímeros como material donor de electrones en la capa absorbente de CSO. 


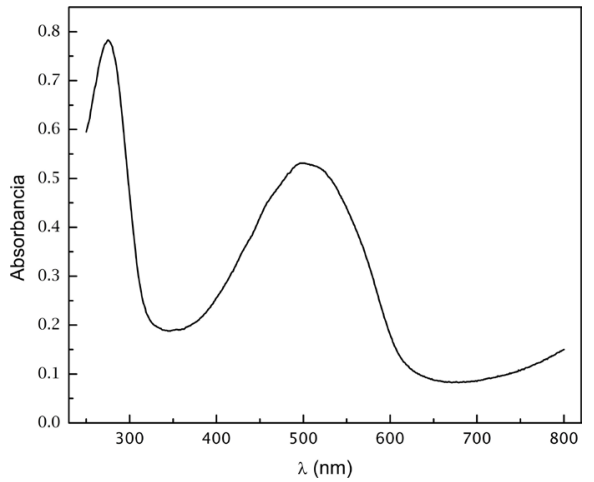

Figura 10. Espectro UV-Vis del poli-3,4-diisobutoxitiofeno.

\subsection{Electropolimerización}

La electropolimerización se llevó acabo con el objetivo de depositar los polímeros directamente sobre el ánodo de la CSO, y desarrollar de esta manera una ruta de fabricación de los dispositivos mucho mas corta; ya que se evitaría la polimerización química, la purificación y la disolución del polímero para depositarlo luego mediante spin coating o alguna otra técnica que utilice tintas o disoluciones del polímero $[15,16,17,18]$.

El único monómero que se pudo electropolímerizar fue el 3,4-dimetoxitiofeno, debido a que el otro (3,4-diisobutoxitiofeno) estaba en bajas concentraciones y presentaba impurezas que no pudieron retirarse por la poca cantidad obtenida de la síntesis.

En esta síntesis electroquímica del polímero se utilizó como electrodo de trabajo una placa de vidrio alcalino recubierto con FTO y con una delgada capa de oro, la cual tuvo que ser depositada para obtener una mayor eficiencia del proceso de electropolimerización.

En la figura 11 se muestra el voltamograma de un ensayo previo de polimerización en el que se aplicó sobre el electrodo de trabajo dos barridos cíclicos de potencial en un rango entre $-500 \mathrm{a}-1300 \mathrm{mV}$. Se observa que la oxidación del monómero ocurre entre 1000 y $1300 \mathrm{mV}$ y el crecimiento del polímero entre -100 a $1000 \mathrm{mV}$.

Del estudio anterior se deduce que el rango de potenciales en el que se debe hacer la electro-polimerización es de -100 hasta $1000 \mathrm{mV}$ (vs. PRE $\mathrm{Ag} / \mathrm{Ag}^{+}$) en el cual no se oxida completamente al monómero, permitiendo así que el catión radical, que es el iniciador, se poduzca y continúe con la reacción de síntesis del polímero [18]. La polimerización culmina al dejar de hacer los barridos anódicos de potencial. La cantidad de polímero producido depende tanto de la forma del potencial aplicado (pulsos, ciclos, etc.) como del rango de potenciales utilizado y del número de barridos o de ciclos. 


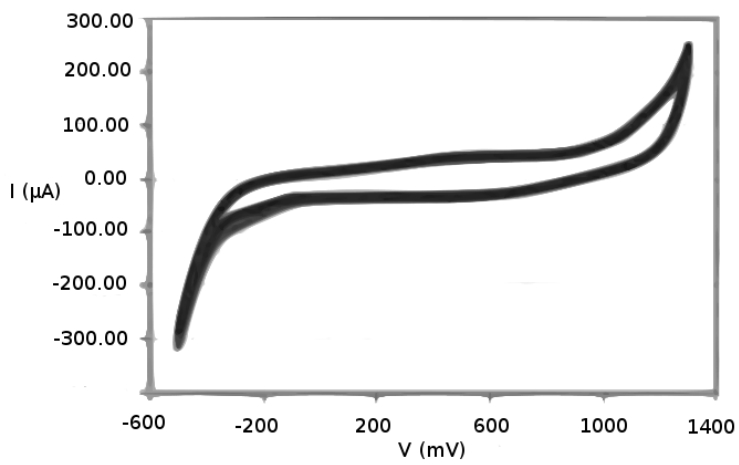

Figura 11. Comportamiento electroquímico, bajo ciclos de potencial del 3,4-dimetoxitiofeno.

En la figura 12 se muestra el voltamperograma de la polimerización electroquímica del compuesto 2. Los potenciales catódicos y anódicos se encuentran dentro del rango que generalmente presentan este tipo de compuestos (200 $\mathrm{mV}-$ $600 \mathrm{mV}$ ) y lo que se observa, el aumento de magnitud de las corrientes anódicas y la disminución de las corrientes catódicas a medida que aumentan los barridos, es un comportamiento similar a la deposición de metales, y corresponde a un crecimiento de la capa de polímero sobre el electrodo [18].

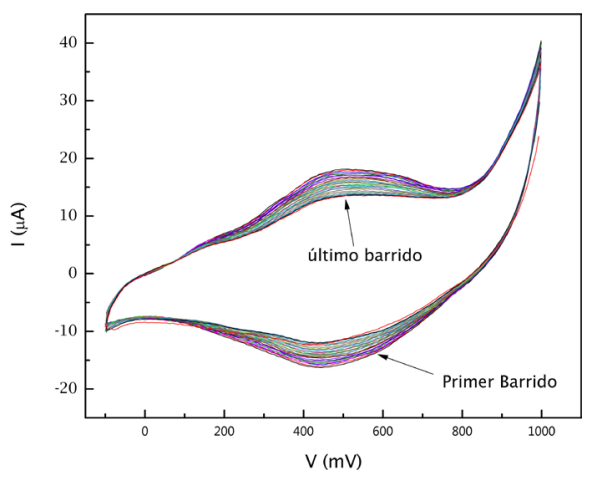

Figura 12. Electropolimerización del 3,4-dimetoxitiofeno, utilizando barridos de potencial.

En la figura 13 se muestran los voltamperogramas correspondientes al estudio del comportamiento electroquímico del poli-3,4-dimetoxitiofeno obtenido tanto por vía electroquímica como por medio de polimerización química utilizando 
el $\mathrm{FeCl}_{3}$. El comportamiento electroquímico del polímero electrosintetizado es completamente diferente a aquel que se observa para el polímero sintetizado por vía química; se aprecian cambios notables de comportamiento tanto para los procesos anódicos como catódicos, los cuales, estan bajo estudio en este momento y los resultados serán publicados próximamente.

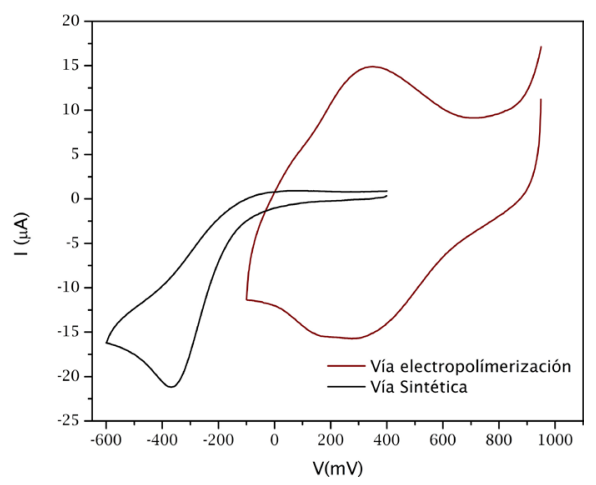

Figura 13. Comportamiento electroquímico del poli-3,4-dimetoxitiofeno, sintetizado mediante las dos rutas electroquímica (ciclos de potencial) y oxidativa, utilizando $\mathrm{FeCl}_{3}$.

De un estudio realizado por medio de microscopía de fuerza atómica (AFM por sus siglas en inglés), cuyos resultados no se muestran aquí, se pudo establecer que el mejor método electroquímico de polimerización es el de aplicación de barridos de potencial; ya que con éste método se obtienen depósitos de poli-3,4dimetoxitiofeno que cubren totalmente el substrato y que son más uniformes que los depósitos obtenidos utilizando pulsos de potencial. En este momento se hacen estudios de electropolimerización sobre electrodos de vidrio recubiertos de ITO en reemplazo del FTO, pues al parecer los polímeros tienen mayor afinidad ITO, y además, no es necesario recubrir el ITO con Au o con Pt para catalizar la electropolimerización de los tiofenos disustituidos $[15,17,18]$.

\section{Conclusiones}

Se realizó la síntesis y caracterización del poli-3,4-dimetoxitiofeno y de una mezcla de poli-3,4-diisobutoxitiofeno/3,4-dimetoxitiofeno, mediante técnicas espectroscópicas de FT-IR, ${ }^{1} \mathrm{H}$ RMN, fluorescencia, UV-VIS. Esta caracterización fue complementada mediante voltamperometría cíclica y microscopía de fuerza atómica.

Los resultados demostraron que al tener band gap bajos, entre 2, 10 y 1,97 eV, y no presentar fluorescencia, los polímeros sintetizados pueden ser utilizados como materiales semiconductores tipo p (donores de electrones) en celdas solares 
orgánicas, lo cual es un avance importante en las investigaciones futuras a desarrollar en el grupo de investigación.

También al observar que hay una electro-polimerización efectiva sobre el mismo sustrato-ánodo se abre la posibilidad de desarrollar, en un futuro cercano, métodos más simples y económicos de fabricación de CSO, que reduzcan el uso de los solventes orgánicos empleados en otros procesos.

\section{Referencias}

1. Krebs, F.C.: Solar Energy Materials \& Solar Cells 83 125-146 (2004)

2. Zhang, S., Wang, J.: J. Mater. Chem., 22, 4178-4187 (2012)

3. Pagliaro, M.: Flexible Solar Cells, Wiley VCH, 31-32, 85-86 p. (2008)

4. Hoppe, H., Sariciftci, N.: J. Mat. Res. vol.19, No.7, 1924-1945 (2004)

5. Spanggaard, H., Krebs, F.: Solar Energy Materials \& solar cells 83 125-146 (2004)

6. Roncali, J.: Chem Rev, 109, 711-738 (2009)

7. McCulloug, R.: Adv. Mat. 10, No 2, 93-116 (1998)

8. Deibel, C., Dyakonov.: Rep. Prog. Phys. 73 096401, 39 (2010)

9. Cheng, Y., et al: Chem Rev, 109, 5868-5923 (2009)

10. Nielsen, C., Bjørnholm, T.: Organic letters, Vol.6, No 19, 3381-3384 (2004)

11. Tripathi, A.K., Sama, J.K., Taneja, S.C.: Indian Journal of Chemistry, Vol.49B, pp 379-381. (2010)

12. Zotti, G., Gallazzi, M.C., Zerbi, G., Meille, S.V.: Synthetic Metals 73 217-225. (1995)

13. Amou, S. et al.: Journal of polymer science: part A: polymer chemistry, vol 37, 1943-1948 (1999)

14. Qi, Z., Wei, B., Sun, Y., Wan, X., Kang, F., Hong, M., Tang, L.: Polym. Bull, (2010)

15. Refczynska, M., Mieczkowski, J., Skompska, M.: Electrochimica Acta 53 2984-993. (2008)

16. Szkurlat, A., Palys, B., Mieczkowski, J., Skompska, M.: Electrochimica Acta 48 3665-3676. (2003)

17. Valaski, R., Canestraro, C.D., Micaroni, L., Mello, R.M.Q., Roman, L.S.: Solar Energy Materials \& Solar Cells 91, 684-688 (2007)

18. Mühlbacher, D., Neugebauer, H., Cravino, A., Sariciftci, N.S., van Duren, J.K.J., Dhanabalan, A., van Hal, P.A., Janssen, R.A.J.: Mol. Cryst. Liq. Cryst., Vol. 385, pp. $205 / 85-212 / 92$. (2002) 